\title{
Diagnostic challenges of single plaque-like lesion paucibacillary leprosy
}

\author{
Raquel Rodrigues Barbieri', Anna Maria Sales², Ximena Illarramendi², Milton Ozório Moraes², \\ José Augusto da Costa Nery' ${ }^{2}$, Suelen Justo Maria Moreira ${ }^{2}$, Euzenir Nunes Sarno ${ }^{2}{ }^{+}$, \\ Alice de Miranda Machado², Fernando Augusto Bozza ${ }^{1}$
}

${ }^{1}$ Instituto Nacional de Infectologia Evandro Chagas ²Laboratório de Hanseníase, Instituto Oswaldo Cruz-Fiocruz, Rio de Janeiro, RJ, Brasil

The diagnosis of single-lesion paucibacillary leprosy remains a challenge. Reviews by expert dermatopathologists and quantitative polymerase chain reaction ( $q P C R$ ) results obtained from 66 single-plaque biopsy samples were compared. Histological findings were graded as high (HP), medium (MP) or low (LP) probability of leprosy or other dermatopathy (OD). Mycobacterium leprae-specific genes were detected using qPCR. The biopsies of 47 out of 57 clinically diagnosed patients who received multidrug therapy were classified as HP/MP, eight of which were qPCR negative. In the LP/OD $(n=19)$, two out of eight untreated patients showed positive qPCR results. In the absence of typical histopathological features, qPCR may be utilised to aid in final patient diagnosis, thus reducing overtreatment and delay in diagnosis.

Key words: paucibacillary leprosy - diagnosis - quantitative polymerase chain reaction

The diagnosis of leprosy is confirmed by evidence of acid-fast bacilli (AFB) in slit-skin smears or tissue samples or by the presence of characteristic histological alterations of the nerves or skin. However, bacilli are rarely found in the paucibacillary (PB) forms of this disease and no gold standard has been established to diagnose cases in which bacilli are not detected (Buhrer-Sekula et al. 2009). Moreover, classical features, such as inflammatory infiltrate within dermal nerves (Ridley 1973), may not be present, rendering histopathological examination inconclusive. Because an accurate diagnosis in these cases is challenging, the results of reviews by expert dermatopathologists and quantitative polymerase chain reaction (qPCR) analyses of single-plaque biopsy samples were compared to evaluate previous therapeutic decisions.

This retrospective study was performed at the Souza Araújo Outpatient Clinic, Leprosy Reference Centre, Oswaldo Cruz Foundation, Rio de Janeiro, Brazil. Skin biopsy samples from 86 patients with a single plaque, but no previous history of leprosy, who attended the clinic for diagnostic purposes from January 2008-December 2011 were selected. Only biopsies obtained from PB patients, i.e., absence of AFB in all of their four-six slit-skin smears, were included. Thus, samples lacking available skin fragments for PCR analysis $(n=18)$ or samples obtained from patients with positive bacterial index results on the slit-skin smear test $(n=2)$ were excluded.

The remaining biopsy samples obtained from 66 patients $(59 \%$ female) with suspected leprosy possessing single plaque-like skin lesions were reviewed by two in-

doi: $10.1590 / 0074-0276140212$

Financial support: CNPq, FAPERJ

+ Corresponding author: euzenir@fiocruz.br

Received 11 June 2014

Accepted 4 September 2014 dependent pathologists. Histological findings were graded as high (HP), medium (MP) or low (LP) probability of leprosy or other dermatopathies (OD) (Fig. 1, Table I).

The presence of Mycobacterium leprae-specific genes was detected using qPCR and the results were compared to the histopathology grading and clinical data. The levels of M. leprae Ag 85B DNA and 16S rDNA in the skin biopsy specimens were estimated using TaqMan qPCR amplification (Life Technologies Corporation, USA) (Martinez et al. 2006, 2009).

Frozen skin biopsies stored in liquid nitrogen were used to isolate DNA. DNA was extracted using a DNeasy Kit (Qiagen Inc, USA) according to the manufacturer's recommendations. All qPCR was performed as previously described (Martinez et al. 2011) and the 85B DNA and $16 \mathrm{~S}$ rDNA were targeted. Briefly, reaction mixtures were prepared in duplicate for each target and exposed to $50^{\circ} \mathrm{C}$ for $2 \mathrm{~min}, 95^{\circ} \mathrm{C}$ for $10 \mathrm{~min}$ and 40 cycles of denaturing for $15 \mathrm{~s}$ and annealing/extension at $60^{\circ} \mathrm{C}$ for $60 \mathrm{~s}$ using a StepOne Real-Time System (Life Technologies). The fluorescence accumulation data were captured by StepOne Sequence Detection System software (Life Technologies) and the $\Delta \mathrm{Rn}$ values were extracted to estimate the cycle threshold $(\mathrm{Ct})$, which was compared to the standard curves constructed using $M$. leprae DNA. The qPCR results were classified as positive or negative. A result was considered positive if the detected amplification resulted in a $\mathrm{Ct}$ value that was lower than at the 38th cycle for at least one amplified target gene.

Statistical Package for the Social Sciences ${ }^{\mathrm{TM}}$ software v.16.0 (USA) was used for data analysis. Categorical variables were analysed using the chi-squared $\left(\chi^{2}\right)$ test at a significance level of $5 \%$. Concordance between the pathologists was determined by calculating the observed agreement and kappa $(\kappa)$ index. $\kappa$ values were interpreted according to a previously described scale (Altman 1991).

This study was conducted in accordance with the norms established by the National Health Council under the Brazilian Ministry of Health (MS/CNS 1996). 
TABLE I

Grading of skin biopsy according to histopathological criteria

Classification
$\begin{aligned} & \text { High probability } \\ & \text { Medium probability }\end{aligned}$
$\begin{gathered}\text { Nerve involvement by inflammatory infiltrate } \\ \text { (Fig. 1A) } \\ \text { Low probability } \\ \text { (Fig. 1B) }\end{gathered}$
$\begin{gathered}\text { Sparse granulomatous inflammatory infiltrate with unidentified nerve bundles } \\ \text { lomatous inflammatory infiltrate and unidentified cutaneous nerves } \\ \text { (Fig. 1C) }\end{gathered}$
Other dermatopathies

(Fig. 1D)

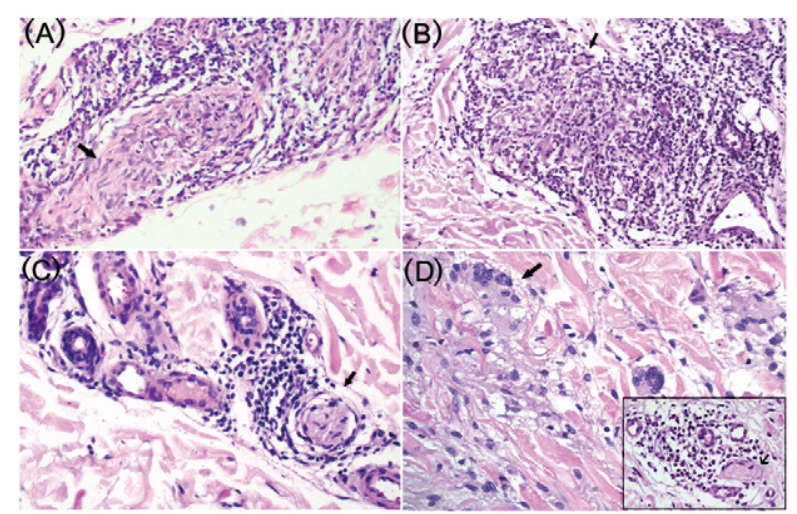

Fig. 1: histopathological findings according to grading (H\&E stained). A: high probability (200X). Epithelioid granuloma invading the dermal nerves (arrow); B: medium probability (100X). Epithelioid granulomas (arrow) without identified nerve bundles in the dermis; C: low probability (200X). Non-specific inflammatory infiltrates around blood vessels, nerve bundles not invaded; D: other dermatopathies (400X). Granuloma annulare. Necrobiosis of collagen fibres and multinucleated cells (arrow); Insert (400X): dermal nerves not invaded (arrowhead).

The Evandro Chagas Research Institute Review Board approved the study protocol (register 62 0059.0.009.00011). All patients signed informed consent forms prior to undergoing routine diagnostic procedures.

On average, the plaques were $5.8 \mathrm{~cm}$ wide and were most frequently $(85 \%)$ located on the upper and lower extremities. Cold thermal hypoaesthesia and anaesthesia in the lesions were found in $98 \%$ of the cases, whereas tactile and pain sensory deficit were present in $75 \%$ and $50 \%$ of the cases, respectively.

The two pathologists were in good agreement with regard to grading classifications. Lymphocytes, epithelioid cells, epithelioid granuloma and superficial as well as deep perivascular infiltrates were the most common findings in the dermis. There was "good" or "very good" agreement between the pathologists with regard to all aforementioned variables. The pathologists were in full agreement for only four items related to inflammatory infiltrate (Table II). After examination by a third pathologist, the final histopathological classification was as follows: $46 \%(\mathrm{n}=30) \mathrm{HP}, 26 \%(\mathrm{n}=17) \mathrm{MP}, 8 \%(\mathrm{n}$ =5) LP and $21 \%(\mathrm{n}=14) \mathrm{OD}$ (Fig. 2). Due to the small number of patients in each group, the cases were grouped together as follows: HP + MP $=47$ and LP $+\mathrm{OD}=19$.

Forty-six (70\%) skin samples were qPCR positive, of which 15 were positive for both assays, 24 were positive only for $16 \mathrm{~S}$ rDNA and seven were positive only for $85 \mathrm{~B}$ DNA. A statistically significant association $\left(\chi^{2}=11.54 ; \mathrm{p}\right.$ $=0.0007)$ was found between the qPCR results and the histopathological classification. Among the 57 clinically diagnosed leprosy patients (indeterminate $=5 \%$, tuberculoid $=22 \%$ and borderline-tuberculoid $=73 \%$ ) who received multidrug therapy (MDT), eight HP/MP cases were qPCR negative. In the LP/OD group, $25 \%$ of the eight untreated patients showed positive qPCR results (Fig. 2). The patients with positive qPCR results who had not received MDT were scheduled for re-evaluation and treatment. Only one patient returned and was treated accordingly.

The absence of a gold standard is a major concern in PB leprosy diagnosis. In addition, the subjectivity inherent in the clinical diagnosis and histopathological analysis of skin lesions may hinder the confirmation of leprosy disease. Analysis by qPCR was used as an additional tool and the results confirmed the leprosy diagnosis in most cases, particularly in patients with HP/MP.

While alteration of sensory evaluation guides the diagnosis of leprosy, it may also be present in other skin pathologies (Gupte et al. 1990, Kumar \& Dogra 2009). Poor agreement between clinical and histologic diagnoses has been previously described (Bhatia et al. 1993).

The difficulty in visualising inflammatory infiltrates within dermal nerve bundles is one of the limitations encountered in the histopathological diagnosis of leprosy in the absence of bacilli (Scollard et al. 2006). Previous studies have also described the value and limitations of histopathology in diagnosing leprosy skin lesions (Sehgal et al. 1977, Bhatia et al. 1993). Disagreement has been reported not only between pathologists, but also between two evaluations by the same pathologist conducted on different occasions (Fine et al. 1993). 
TABLE II

Frequency of pathological findings and agreement between two pathologists as calculated by the kappa ( $\kappa)$ coefficient

\begin{tabular}{|c|c|c|c|}
\hline & $\begin{array}{c}\text { Pathologist } 1 \\
\text { n (\%) }\end{array}$ & $\begin{array}{c}\text { Pathologist } 2 \\
\text { n (\%) }\end{array}$ & $\kappa$ coefficient \\
\hline \multicolumn{4}{|l|}{ Epidermal findings } \\
\hline Rectification of ridges & $23(35)$ & $20(30)$ & 0.35 \\
\hline Erosion of the basal layer & $18(27)$ & $10(15)$ & 0.64 \\
\hline Exocytosis of lymphocytes & $35(53)$ & $39(59)$ & 0.51 \\
\hline Parakeratosis & $9(14)$ & $7(11)$ & 0.86 \\
\hline Hyperkeratosis & $9(14)$ & $7(11)$ & 0.43 \\
\hline Spongiosis & $7(11)$ & $3(5)$ & 0.15 \\
\hline Acanthosis & $10(15)$ & $9(14)$ & 0.94 \\
\hline \multicolumn{4}{|l|}{ Infiltrate composition } \\
\hline Lymphocytes & $66(100)$ & $66(100)$ & 1 \\
\hline Macrophages & $64(97)$ & $32(49)$ & -0.002 \\
\hline Epithelioid cells & $46(70)$ & $50(76)$ & 0.7 \\
\hline Plasmocytes & $7(11)$ & $9(14)$ & 0.29 \\
\hline Neutrophils & $3(5)$ & $2(3)$ & 0.38 \\
\hline Eosinophils & $4(6)$ & $1(2)$ & 0.38 \\
\hline \multirow[t]{2}{*}{ Giant cells } & $22(33)$ & $19(29)$ & 0.54 \\
\hline & $7(11)$ & $1(2)$ & -0.03 \\
\hline \multicolumn{4}{|l|}{ Infiltrate architecture } \\
\hline No granuloma & $21(32)$ & $21(32)$ & 0.66 \\
\hline Epithelioid granuloma & $45(68)$ & $45(68)$ & 1 \\
\hline \multicolumn{4}{|l|}{ Infiltrate location } \\
\hline Superficial dermis & $66(100)$ & $66(100)$ & 1 \\
\hline Deep dermis & $54(82)$ & $53(80)$ & 0.95 \\
\hline Perivascular & $66(100)$ & $66(100)$ & 1 \\
\hline Perineural & $41(62)$ & $31(47)$ & 0.52 \\
\hline Periglandular & $34(52)$ & $50(76)$ & 0.32 \\
\hline Hair erector muscle & $9(14)$ & $11(17)$ & 0.53 \\
\hline \multicolumn{4}{|l|}{ Dermal Nerves } \\
\hline Preserved & $19(29)$ & $12(18)$ & 0.54 \\
\hline Unidentified & $17(26)$ & $26(39)$ & 0.5 \\
\hline Infiltrated & $30(46)$ & $26(39)$ & 0.57 \\
\hline \multicolumn{4}{|l|}{ Dermal findings } \\
\hline Necrosis & $8(12)$ & $4(6)$ & 0.64 \\
\hline
\end{tabular}

values demonstrating high concordance between pathologists are in bold.

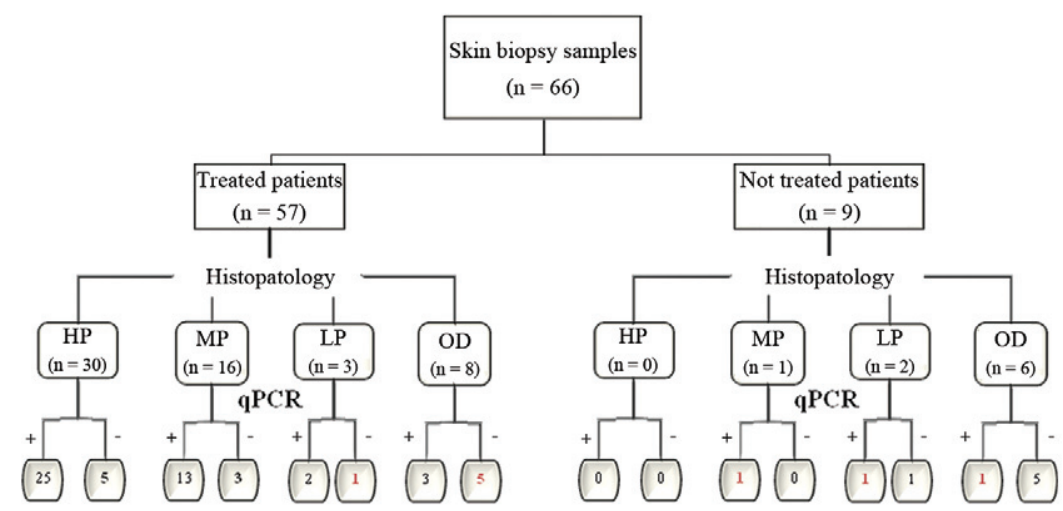

Fig. 2: flow chart of the relationship between histopathology, quantitative polymerase chain reaction (qPCR) results and treatment decisions according to previous clinical and histopathological diagnoses. The incongruent cases regarding results and clinical management are highlighted. HP: high probability; LP: low probability; MP: medium probability; OD: other dermatopathies. 
The advent of real-time qPCR has improved the specificity and sensitivity of the diagnosis of early forms of this disease compared to conventional PCR techniques (Rudeeaneksin et al. 2008, Martinez et al. 2011), which have produced consistent false-negative results in the detection of other diseases such as psoriasis, sarcoidosis and leishmaniasis (Rudeeaneksin et al. 2008, Lini et al. 2009). The sensitivity of qPCR has been found to be over $60 \%$ for the identification of both 85B DNA and 16S rDNA (Martinez et al. 2011). Because we combined both assays to increase positivity, we considered the two LP/OD cases with positive qPCR results to be underdiagnosed, while the one LP and five OD cases who received $\mathrm{WHO} / \mathrm{MDT}$ most likely received unnecessary MDT based on the qPCR results. If qPCR is considered to be the gold-standard test in PB leprosy, these six patients could be considered overdiagnosed.

Bacilli clearance induced by the host immunological response reduces DNA availability in tissues, falling below the threshold for detection by molecular methods. Despite this limitation, the combined qPCR results were able to confirm the leprosy diagnosis in $70 \%$ of all cases.

The use of additional diagnostic techniques when histopathology is inconclusive could ensure early diagnosis, thus enabling leprosy patients to receive timely treatment and avoid sequelae. For patients with other skin disorders, a correct diagnosis would both prevent the onset of complications related to unnecessary treatment and avoid the stigma often associated with leprosy. Lastly, a more accurate diagnosis could provide better surveillance data and improved disease control strategies.

\section{ACKNOWLEDGEMENTS}

To the team of dermatologists, nurses and technicians that collaborate at the Souza Araújo Outpatient Clinic and the Leprosy Laboratory and to Dr Bernard Kac and Judy Grevan, for English editing.

\section{REFERENCES}

Altman DG 1991. Practical statistics for medical research, Chapman and Hall, London, 611 pp.

Bhatia AS, Katoch K, Narayanan RB, Ramu G, Mukherjee A, Lavania RK 1993. Clinical and histopathological correlation in the classification of leprosy. Int J Lepr Other Mycobact Dis 61: 433-438.
Buhrer-Sekula S, Illarramendi X, Teles RB, Penna ML, Nery JA, Sales AM, Oskam L, Sampaio EP, Sarno EN 2009. The additional benefit of the ML Flow test to classify leprosy patients. Acta Trop 111: $172-176$

Fine PE, Job CK, Lucas SB, Meyers WM, Ponnighaus JM, Sterne JA 1993. Extent, origin and implications of observer variation in the histopathological diagnosis of suspected leprosy. Int J Lepr Other Mycobact Dis 61: 270-282.

Gupte MD, Vallishayee RS, Nagaraju B, Ramalingam A, Lourdusamy G, Kannan S 1990. Inter-observer agreement and clinical diagnosis of leprosy for prophylaxis studies. Indian J Lepr 62: 281-295.

Kumar B, Dogra S 2009. Leprosy: a disease with diagnostic and management challenges! Indian J Dermatol Venereol Leprol 75: 111-115.

Lini N, Shankernarayan NP, Dharmalingam K 2009. Quantitative real-time PCR analysis of Mycobacterium leprae DNA and mRNA in human biopsy material from leprosy and reactional cases. J Med Microbiol 58: 753-759.

Martinez AN, Britto CF, Nery JA, Sampaio EP, Jardim MR, Sarno EN, Moraes MO 2006. Evaluation of real-time and conventional PCR targeting complex 85 genes for detection of Mycobacterium leprae DNA in skin biopsy samples from patients diagnosed with leprosy. J Clin Microbiol 44: 3154-3159.

Martinez AN, Lahiri R, Pittman TL, Scollard D, Truman R, Moraes MO, Williams DL 2009. Molecular determination of Mycobacterium leprae viability by use of real-time PCR. J Clin Microbiol 47: 2124-2130.

Martinez AN, Ribeiro-Alves M, Sarno EN, Moraes MO 2011. Evaluation of qPCR-based assays for leprosy diagnosis directly in clinical specimens. PLoS Negl Trop Dis 5: e1354.

MS/CNS - Ministério da Saúde Brasil/Conselho Nacional de Saúde 1996. Diretrizes e normas de pesquisa em seres humanos. Resolução 196/96, 9/10/96, DOU 16/10/96:21081-21085.

Ridley DS 1973. The pathogenesis of the early skin lesion in leprosy. J Pathol 111: 191-206.

Rudeeaneksin J, Srisungngam S, Sawanpanyalert P, Sittiwakin T, Likanonsakul S, Pasadorn S, Palittapongarnpim P, Brennan PJ, Phetsuksiri B 2008. LightCycler real-time PCR for rapid detection and quantitation of Mycobacterium leprae in skin specimens. FEMS Immunol Med Microbiol 54: 263-270.

Scollard DM, Adams LB, Gillis TP, Krahenbuhl JL, Truman RW, Williams DL 2006. The continuing challenges of leprosy. Clin Microbiol Rev 19: 338-381.

Sehgal VN, Rege VL, Reys M 1977. Correlation between clinical and histopathologic classification in leprosy. Int J Lepr Other Mycobact Dis 45: 278-280. 